\title{
Existence of Resonances in Three Dimensions
}

\author{
Antônio Sá Barreto ${ }^{1}$, Maciej Zworski ${ }^{2}$ \\ ${ }^{1}$ Department of Mathematics, Purdue University, West Lafayette, IN 47907, USA \\ 2 Department of Mathematics, The Johns Hopkins University, Baltimore, MD 21218, USA
}

Received: 26 September 1994/in revised form: 23 December 1994

\begin{abstract}
If $P$ is an elliptic self-adjoint perturbation of the Laplacian $\Delta$ on $\mathbb{R}^{3}$, and the coefficients of $P-\Delta$ decay super-exponentially, then we show that $P$ has infinitely many resonances. The resonances are defined here as the poles of the meromorphic continuation of $\left(P-\lambda^{2}\right)^{-1}$.
\end{abstract}

\section{Introduction and Statement of Results}

The purpose of this note is to show the existence of infinitely many resonances for super-exponentially decaying elliptic self-adjoint perturbations of the Laplacian in $\mathbb{R}^{3}$.

The key component is the extension of Melrose's Poisson formula to that class of perturbations:

$$
\operatorname{tr}\left(U(t)-U_{0}(t)\right)=\sum_{\text {resonances }} e^{-l \lambda_{j} t}, \quad t>0,
$$

where $U(t)$ denotes the perturbed wave group and $U_{0}(t)$ the free one and where we included square roots of eigenvalues among resonances. The formula (1.1) was proved for compactly supported potential and obstacle scattering by Melrose $[9,10]$ and was then extended to more general compactly supported perturbations in [18]. It was used by Sjöstrand and the second author [17,18] and then by Farhy [4] and Vodev [22] to obtain lower bounds on the number of scattering poles in a variety of situations, always using the strong singularities of the wave group. It is obvious however that non-vanishing of the wave trace for $t>0$ already guarantees the existence of resonances or eigenvalues. That non-vanishing can be immediately inferred from the non-vanishing of the higher heat/wave coefficients, that it is those which do not correspond to $\delta$-functions at $t=0$. As was pointed out to us by Melrose, the vanishing of the zeroth coefficient in odd dimensions implies that the number of resonances is infinite (see Proposition 4.3 of [12] for consequences of that for scattering by compactly supported potentials in odd dimensions) but unlike in $[17,18,4,22]$ this does not seem to lead to quantitative information. 


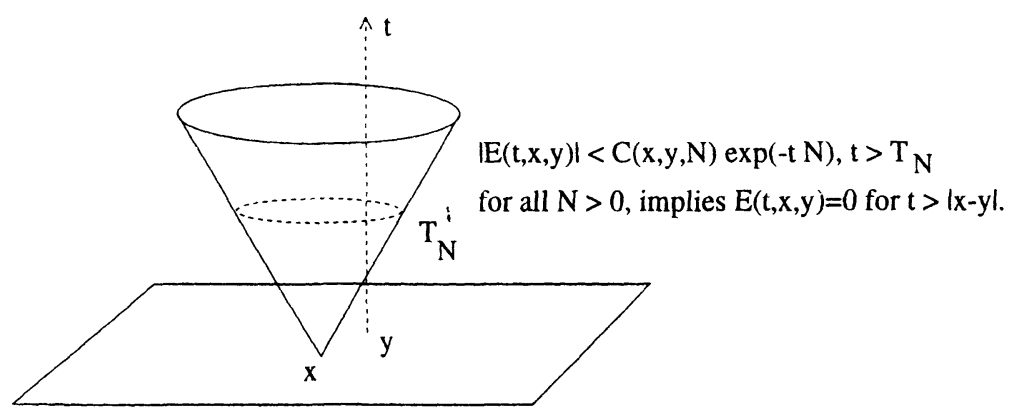

Fig. 1. Decay of the fundamental solution

Our motivation came also from a question concerning the fundamental solution of the perturbed wave equation in $\mathbb{R}^{n}, n$ odd: does a super-exponential decay of the fundamental solution inside of light cones imply its vanishing there? For self-adjoint elliptic perturbations and $n=3$ this is answered affirmatively in the Corollary below. In some sense this is a unique continuation theorem at $t=\infty$ and it motivates also the super-exponential decay assumption for the perturbation - at the moment however we need to assume that the decay is uniform, $\mathcal{O}\left(\exp \left(-A|x|^{1+\varepsilon}\right)\right)$ for fixed $\varepsilon>0$.

From the scattering theory point of view the super-exponential decay assumption is reasonable as it guarantees a meromorphic continuation of the resolvent to $\mathbb{C}$ and thus a global definition of resonances. It is also natural if infinitely many resonances are to be expected: in one dimension there exist potentials of exact exponential decay and with finitely many resonances (see for instance [3] for the computation of the scattering matrix for that type of potentials).

Let $C_{b}^{\infty}\left(\mathbb{R}^{n}\right)$ denote the space of $C^{\infty}$ functions with bounded derivatives. We can state the application of the present method as

Theorem. Let $P$ be an elliptic self-adjoint operator on $L^{2}\left(\mathbb{R}^{3}, \sqrt{\bar{g}} d x\right)$ :

$$
P=-\frac{1}{\sqrt{g}} \sum_{i, j=1}^{3} \partial_{x_{i}} \sqrt{\bar{g}} g^{i j} \partial_{x_{j}}+V, \quad \bar{g}=\operatorname{det}\left(g_{l j}\right), \quad\left(g^{i j}\right)=\left(g_{i j}\right)^{-1},
$$

where $V, g_{i,} \in C_{b}^{\infty}\left(\mathbb{R}^{3}\right)$ satisfy for some fixed $\varepsilon>0$ and $A, B$,

$$
|V(x)| \leqq B e^{-A|x|^{1+\varepsilon}}, \quad \text { and } \quad\left|g_{\imath \jmath}(x)-\delta_{\imath j}\right| \leqq B e^{-A|x|^{1+\varepsilon}}, \quad 1 \leqq i, j \leqq 3 .
$$

If $V \neq 0$ or the metric $g_{l j}$ is not flat then the meromorphic continuation of $\left(P-\lambda^{2}\right)^{-1}$ has infinitely many poles.

Expressing the resolvent in terms of the wave group immediately gives

Corollary. Let $P$ be as in the Theorem above and let $E(t, x, y)$ be the fundamental solution for the wave equation:

$$
\left(D_{t}^{2}-P\right) E(t, x, y)=0, \quad E(0, x, y)=0, \quad \partial_{t} E(0, x, y)=\delta(x-y) .
$$

If $\forall x, y \in \mathbb{R}^{3}$ and $\forall N \in \mathbb{N} \exists T, C$ such that $\forall t>T|E(t, x, y)| \leqq C e^{-N t}$, then $P=\Delta$. 
We remark that the argument used here is similar to that in the proof of Corollary 25 of [1] where the analytic Riemannian three manifolds for which the strong Huyghens principle holds are characterized to be flat. We note that a slight extension of computation of [1] in Lemma 6 below shows the same conclusion for Schrödinger operators on compact three manifolds, except for the locally symmetric case and $\Delta_{G}+\frac{1}{6} \tau$, where $\tau$ is the (constant) scalar curvature.

\section{Upper Bounds on the Number of Resonances for Non-Compactly Supported Perturbations}

To generalize the Poisson summation formula to our case we need a uniform polynomial bound on the number of resonances for non-compactly supported perturbations. Here we will only consider elliptic self-adjoint operators in $\mathbb{R}^{n}, n$ odd. Thus we define

$$
\begin{gathered}
P=-\sum \partial_{x_{i}} a_{i j} \partial_{x,}+V, a_{l \jmath} \in L^{\infty}\left(\mathbb{R}^{n}\right) \cap \operatorname{Lip}\left(\mathbb{R}^{n}\right), \\
V \in L^{\infty}\left(\mathbb{R}^{n}\right),\left(a_{l j}\right) \gg c_{0} I, \quad c_{0}>0, \\
e^{A|x|^{1+t}}\left|a_{i j}-\delta_{l j}(x)\right| \leqq B, \quad e^{A|x|^{1+i}}|V(x)| \leqq B
\end{gathered}
$$

where $\varepsilon, A, B$ are fixed.

The regularity assumptions in this section are considerably weaker than in the Theorem above since all that is needed here is $(2.2)$ and the boundedness $(P+i)^{-1}$ : $L^{2}\left(\mathbb{R}^{n}\right) \rightarrow H^{2}\left(\mathbb{R}^{n}\right)$.

The meromorphic continuation of the resolvent is essentially well-known but before presenting its proof we need two lemmas. We denote by $R$ and $R_{0}$ the resolvent of $P$ and $\Delta=-\sum \partial_{x_{i}}^{2}$, respectively,

$$
\begin{gathered}
R(\lambda)=\left(P-\lambda^{2}\right)^{-1}: L^{2}\left(\mathbb{R}^{n}\right) \rightarrow H^{2}\left(\mathbb{R}^{n}\right), \quad \operatorname{Im} \lambda>0 \quad \lambda^{2} \notin \sigma(P), \\
R_{0}(\lambda)=\left(\Delta-\lambda^{2}\right)^{-1}: L^{2}\left(\mathbb{R}^{n}\right) \rightarrow H^{2}\left(\mathbb{R}^{n}\right), \operatorname{Im} \lambda>0 .
\end{gathered}
$$

recalling that for $n$ odd $R_{0}(\lambda)$ extends to an entire family of operators $e^{-\langle x\rangle^{1+\delta}} L^{2}\left(\mathbb{R}^{n}\right) \rightarrow e^{\langle x\rangle^{1+\delta}} H^{2}\left(\mathbb{R}^{n}\right), \delta>0$, where $\langle x\rangle=\left(1+|x|^{2}\right)^{\frac{1}{2}}$.

For $N \in \mathbb{R}$ we define

$$
\chi_{N}(x)=\chi\left(|x| /\left(C|N|^{\frac{1}{r}}\right)\right) \text { and }\langle x\rangle_{N} \stackrel{\text { def }}{=} \chi_{N}(x)\left(1+|x|^{2}\right)^{\frac{1}{2}},
$$

where $C$ is large, $\chi \in C^{\infty}(\mathbb{R}), 0 \leqq \chi \leqq 1, \chi(t)=0$ for $t<1$ and $\chi(t)=1$ for $t>2$. We use this to define weighted $L^{2}$ spaces

$$
L_{N}^{2}\left(\mathbb{R}^{n}\right) \stackrel{\text { def }}{=} e^{N\langle x\rangle_{N}} L^{2}\left(\mathbb{R}^{n}\right) .
$$

We now have:

Lemma 1. For $\operatorname{Im} \lambda \geqq 0, n$ odd,$N>0$ and $|\lambda| \leqq C N$,

$$
R_{0}(\lambda)=\mathcal{O}_{\varepsilon}\left(N^{\frac{1}{\varepsilon}} /(1+|\lambda|)\right): L_{-N}^{2}\left(\mathbb{R}^{n}\right) \rightarrow L_{N}^{2}\left(\mathbb{R}^{n}\right),
$$


and for $\operatorname{Im} \lambda>-N+\gamma, \gamma>0$ we have

$$
\begin{gathered}
R_{0}(\lambda)=\mathcal{O}_{\varepsilon, N, \gamma}(1): L_{-N}^{2}\left(\mathbb{R}^{n}\right) \rightarrow L_{N}^{2}\left(\mathbb{R}^{n}\right), \\
e^{-N\langle x\rangle_{N}} R_{0}(\lambda)=\mathcal{O}_{\varepsilon, N, \gamma, \lambda}(1): L_{-N}^{2}\left(\mathbb{R}^{n}\right) \rightarrow H^{2}\left(\mathbb{R}^{n}\right) .
\end{gathered}
$$

Proof. We define $\chi_{N}^{1}(x)=\chi\left(|x| /\left(\tilde{C}|N|^{\frac{1}{\varepsilon}}\right)\right)$ so that $\chi_{N} \equiv 1$ on supp $\chi_{N}^{1}$. We then rewrite the reduced kernel as

$$
\begin{aligned}
e^{-N\langle x\rangle_{N}} R_{0}(\lambda) e^{-N\langle x\rangle_{N}}= & \chi_{N}^{1} e^{-N\langle x\rangle_{N}} R_{0}(\lambda) e^{-N\langle x\rangle_{N}} \chi_{N}^{1} \\
& +\left(1-\chi_{N}^{1}\right) e^{-N\langle x\rangle_{N}} R_{0}(\lambda) e^{-N\langle x\rangle_{N}}\left(1-\chi_{N}^{1}\right) \\
& +\left(1-\chi_{N}^{1}\right) e^{-N\langle x\rangle_{N}} R_{0}(\lambda) e^{-N\langle x\rangle_{N}} \chi_{N}^{1} \\
& +\chi_{N}^{1} e^{-N\langle x\rangle_{N}} R_{0}(\lambda) e^{-N\langle x\rangle_{N}}\left(1-\chi_{N}^{1}\right) .
\end{aligned}
$$

To estimate the norms we write for $\operatorname{Im} \lambda \geqq 0$,

$$
R_{0}(\lambda)=\frac{i}{\lambda} \int_{0}^{\infty} \cos (t \sqrt{\Delta}) e^{i \lambda t} d t
$$

Since $\chi_{N}^{1} e^{-N\langle x\rangle_{N}}=\chi_{N}^{1} e^{-N\langle x\rangle}$, the norm of the first term is bounded by the norm of $e^{-N\langle x\rangle} R_{0}(\lambda) e^{-N\langle x\rangle}$. Let $\psi \in C_{0}^{\infty}(\mathbb{R})$ satisfy $0 \leqq \psi \leqq 1, \psi=1$ in $|x|<1$ and $\psi=$ 0 in $|x|>2$. Then (2.7) and the sharp Huyghens principle show that the operator with the kernel $\psi\left(|x-y|^{2}\right) R_{0}(\lambda)(x, y)$ is $\mathcal{O}\left((1+|\lambda|)^{-1}\right): L^{2}\left(\mathbb{R}^{n}\right) \rightarrow L^{2}\left(\mathbb{R}^{n}\right)$. Since that eliminates the singularities at $x=y$, Schur's lemma gives the same bound for the operator with the kernel

$$
e^{-N\langle x\rangle}(1-\psi)\left(|x-y|^{2}\right) R_{0}(\lambda)(x, y) e^{-N\langle y\rangle},
$$

since in fact it gives the norm $\mathcal{O}\left(N^{-1}\right)$. For the second term in (2.6) we note that by the finite speed of propagation the integration can be restricted to $\left[0, C^{\prime} N^{\frac{1}{8}}\right]$ and thus the norm is bounded by $C^{\prime} N^{\frac{1}{\varepsilon}} /(1+|\lambda|)$.

To estimate the norm of the remaining two terms we consider the Schwartz kernels:

$$
\left(1-\chi_{N}^{1}(x)\right) e^{-N\langle x\rangle_{N}} \cos (t \sqrt{\Delta})(x, y) e^{-N\langle y\rangle} \chi_{N}^{1}(y)
$$

which vanishes unless $t=|x-y|$, that is $t=|y|+\mathcal{O}\left(N^{\frac{1}{\varepsilon}}\right)$. Hence

$$
\left\|\left(1-\chi_{N}^{1}\right) e^{-N\langle x\rangle_{N}} \cos (t \sqrt{\Delta}) e^{-N\langle x\rangle} \chi_{N}^{1}\right\|_{L^{2} \rightarrow L^{2}} \leqq e^{-N\left(t-C N^{\frac{1}{\varepsilon}}\right)_{+}},
$$

where $z_{+}=z$ for $z \geqq 0$ and $z_{+}=0$ for $z<0$. Thus

$$
\left\|\left(1-\chi_{N}^{1}\right) e^{-N\langle x\rangle_{N}} R_{0}(\lambda) e^{-N\langle x\rangle_{N}} \chi_{N}^{1}\right\|_{L^{2}-L^{2}}=\mathcal{O}\left(N^{\frac{1}{\varepsilon}} /(1+|\lambda|)\right),
$$

with the same estimate for the fourth term.

Since $L_{ \pm N}^{2} \simeq e^{ \pm N\langle x\rangle} L^{2}\left(\mathbb{R}^{n}\right)$, (2.4) is immediate but now the norm estimates depend on $N$ (as they do in the identification above). For (2.5) we use $\Delta R_{0}(\lambda)=$ $\left(I+\lambda^{2}\right) R_{0}(\lambda)$ and (2.4). 
Lemma 2. For $\lambda_{0} \in i \mathbb{R}_{+},\left|\lambda_{0}\right| \geqq C_{1} N \gg c$ with $C_{1}$ sufficiently large,

$$
\begin{gathered}
e^{c\langle x\rangle} R\left(\lambda_{0}\right) e^{-c\langle x\rangle}=\mathcal{O}_{\varepsilon, c}\left(N^{-2}\right): L_{-N}^{2}\left(\mathbb{R}^{n}\right) \rightarrow L_{-N}^{2}\left(\mathbb{R}^{n}\right), \\
e^{N\langle x\rangle_{N}} R\left(\lambda_{0}\right)=\mathcal{O}_{\varepsilon}(1): L_{-N}^{2}\left(\mathbb{R}^{n}\right) \rightarrow H^{2}\left(\mathbb{R}^{n}\right) .
\end{gathered}
$$

The norm estimate on (2.9) depends in $\varepsilon, A, B$ in (2.2), Lipschitz constants of $a_{i j}$ 's and $c_{0}$ in (2.1).

Proof. We briefly recall the argument from [15] and noting that $\left|\nabla\langle x\rangle_{N}\right| \leqq C$ we start by writing

$$
e^{N\langle x\rangle_{N}+c\langle x\rangle}\left(P-\lambda_{0}^{2}\right) e^{-N\langle x\rangle_{N}-c\langle x\rangle}=P+N A_{N}+N^{2} c_{N}-\lambda_{0}^{2}-N^{2},
$$

where $A_{N}$ is a first order operator with uniformly bounded coefficients and $c_{N}$ is a smooth bounded function. Since $R(\mu)=\mathcal{O}\left(|\mu|^{-1-k}\right): L^{2}\left(\mathbb{R}^{n}\right) \rightarrow H^{1-k}\left(\mathbb{R}^{n}\right), k=$ 0,1 , for $\mu \in i \mathbb{R}_{+}$, we have for $-\lambda_{0}^{2}-N^{2} \gg N^{2}$,

$P+N A_{N}+N^{2} c_{N}-\left(\lambda_{0}^{2}+N^{2}\right)=\left(I+\left(N A_{N}+N^{2} c_{N}\right) R\left(i\left|\lambda_{0}^{2}+N^{2}\right|^{\frac{1}{2}}\right)\right)\left(P-\left(\lambda_{0}^{2}+N^{2}\right)\right)$,

where the first factor on the right-hand side is invertible on $L^{2}\left(\mathbb{R}^{n}\right)$. Hence the conjugated operator $(2.10)$ is invertible on $L^{2}\left(\mathbb{R}^{n}\right)$ with norm $\mathcal{O}\left(N^{-2}\right)$ and we obtain (2.8).

For (2.9) we integrate by parts and use (2.1) to get

$$
\left\langle P R\left(\lambda_{0}\right) u, R\left(\lambda_{0}\right) u\right\rangle_{L_{-N}^{2}} \geqq C_{3}\left\|\nabla R\left(\lambda_{0}\right) u\right\|_{L_{-N}^{2}}^{2}-C_{2} N\left\langle\nabla R\left(\lambda_{0}\right) u, R\left(\lambda_{0}\right) u\right\rangle_{L_{-N}^{2}} .
$$

From (2.8) we obtain that

$$
e^{N\langle x\rangle_{N}} \nabla R\left(\lambda_{0}\right)=\mathcal{O}\left(N^{-1}\right): L_{-N}^{2}\left(\mathbb{R}^{n}\right) \rightarrow L^{2}\left(\mathbb{R}^{n}\right)
$$

Next we observe that for some $B_{N} \in \operatorname{Diff}^{1}\left(\mathbb{R}^{n}\right)$ with uniformly bounded coefficients,

$$
P e^{N\langle x\rangle_{N}} R\left(\lambda_{0}\right)=e^{N\langle x\rangle_{N}}\left(\mathcal{O}\left(N^{2}\right) R\left(\lambda_{0}\right)+\mathcal{O}(N) B_{N} R\left(\lambda_{0}\right)+\left(I+\lambda_{0}^{2}\right) R\left(\lambda_{0}\right)\right) .
$$

Thus if $u \in L_{-N}^{2}\left(\mathbb{R}^{n}\right)$ one obtains that $e^{N\langle x\rangle_{N}} R\left(\lambda_{0}\right) u \in L^{2}\left(\mathbb{R}^{n}\right)$ and $P e^{N\langle x\rangle_{N}} R\left(\lambda_{0}\right) u \in$ $L^{2}\left(\mathbb{R}^{n}\right)$. Hence (2.9) follows, as $P$ is uniformly elliptic.

Proposition 1. For $P$ satisfying (2.1) and (2.2) the resolvent $\left(P-\lambda^{2}\right)^{-1}: L^{2}\left(\mathbb{R}^{n}\right) \rightarrow$ $H^{2}\left(\mathbb{R}^{n}\right), \operatorname{Im} \lambda>0, \lambda^{2} \notin \sigma(P)$, has a meromorphic continuation

$$
R(\lambda): e^{-\langle x\rangle^{1+\delta}} L^{2}\left(\mathbb{R}^{n}\right) \rightarrow e^{\langle x\rangle^{1+\delta}} H^{2}\left(\mathbb{R}^{n}\right),
$$

for any $\delta>0$. The poles are of finite rank and their multiplicity

$$
\text { multiplicity of } \lambda_{0}=\operatorname{rank} \int_{\partial D\left(\lambda_{0}, \varepsilon\right)} R(\lambda) \lambda d \lambda
$$

is independent of $\delta$.

Proof. We write $Q=P-\Delta$. Thus for $\operatorname{Im} \lambda>0, \lambda^{2} \notin \sigma(P)$,

$$
R(\lambda)=R_{0}(\lambda)-R(\lambda) Q R_{0}(\lambda),
$$


which using the resolvent identity $R(\lambda)=R\left(\lambda_{0}\right)+\left(\lambda^{2}-\lambda_{0}^{2}\right) R(\lambda) R\left(\lambda_{0}\right)$ gives

$$
R(\lambda)\left(I+\left(\lambda^{2}-\lambda_{0}^{2}\right) R\left(\lambda_{0}\right) Q R_{0}(\lambda)\right)=R_{0}(\lambda)-R\left(\lambda_{0}\right) Q R_{0}(\lambda) .
$$

To obtain meromorphic continuation we follow the standard application of Fredholm theory once we show that $R\left(\lambda_{0}\right) Q R_{0}(\lambda)$ is compact from $L_{-N}^{2}\left(\mathbb{R}^{n}\right)$ to itself. Since

$$
Q e^{N\langle x\rangle_{N}}=\mathcal{O}_{N}(1): H^{2}\left(\mathbb{R}^{n}\right) \rightarrow L_{-M}^{2}\left(\mathbb{R}^{n}\right)
$$

for any $M$ (with norm independent of $M$ ), if $|\lambda|<C N, \operatorname{Im} \lambda>-N+\gamma$ then (2.5) shows that

$$
Q R_{0}(\lambda): L_{-N}^{2}\left(\mathbb{R}^{n}\right) \rightarrow L_{-M}^{2}\left(\mathbb{R}^{n}\right)
$$

for any $M$. If we choose $\lambda_{0}$ as in Lemma 2 with $\left|\lambda_{0}\right| \gg 2 M>2 N$ then by (2.9),

$$
R\left(\lambda_{0}\right): L_{-M}^{2}\left(\mathbb{R}^{n}\right) \rightarrow e^{-M\langle x\rangle_{M}} H^{2}\left(\mathbb{R}^{n}\right),
$$

so that

$$
\left(\lambda^{2}-\lambda_{0}^{2}\right) R\left(\lambda_{0}\right) Q R_{0}(\lambda): L_{-N}^{2}\left(\mathbb{R}^{n}\right) \rightarrow e^{-M\langle x\rangle_{M}} H^{2}\left(\mathbb{R}^{n}\right) \hookrightarrow L_{-N}^{2}\left(\mathbb{R}^{n}\right),
$$

with the last inclusion compact since $M>N$. Putting $\lambda=\lambda_{0}$ (which is possible as long as $\left.\left|\lambda_{0}\right| \leqq C N\right)$ we obtain the invertibility of $I+\left(\lambda^{2}-\lambda_{0}^{2}\right) R\left(\lambda_{0}\right) Q R_{0}(\lambda)$ at one point and by the compactness of the second term we obtain a meromorphic inverse from $L_{-N}^{2}\left(\mathbb{R}^{n}\right)$ to $L_{-N}^{2}\left(\mathbb{R}^{n}\right)$ if $\operatorname{Im} \lambda>-N+\gamma$. Hence for $\operatorname{Im} \lambda>-N+\gamma$ we established the meromorphy of

$$
R(\lambda): L_{-N}^{2}\left(\mathbb{R}^{n}\right) \rightarrow L_{N}^{2}\left(\mathbb{R}^{n}\right) .
$$

Since the ranks of the poles are finite and since the polar parts at $\lambda_{0}$ are in $L_{N}^{2}\left(\mathbb{R}^{n}\right) \otimes$ $L_{N}^{2}\left(\mathbb{R}^{n}\right), N>-\operatorname{Im} \lambda_{0}$ the independence of the multiplicity of $N$ and $\delta$ follows.

Proposition 2. Let $N(r)$ be the number of poles of $R(\lambda)$ in $\{\zeta:|\zeta| \leqq r\}$ counted with their multiplicities. Then there exists a constant $C_{\delta}$ depending only on $\delta>$ $0, \varepsilon, A, B$ in (2.2), $c_{0}$ in (2.1) and the Lipschitz constant of the coefficients of $P$ such that for any $\delta>0$,

$$
N(r) \leqq C_{\delta} r^{n\left(\max \left(\frac{1+\varepsilon}{\varepsilon}, \frac{3}{2}+\frac{1}{2 \varepsilon}+\delta\right)\right)} .
$$

Proof. We apply a modified version of the Fredholm determinant argument of $[10,11,24,19,20]$ - see also [25 and 6]. Thus we start by estimating the characteristic values in the good and bad half planes, $\operatorname{Im} \lambda \geqq 0$ and $\operatorname{Im} \lambda \leqq 0$ respectively. More precisely for any large $N$ we choose $\lambda_{0} \in i \mathbb{R}_{+}, 2 C_{1} N \geqq\left|\lambda_{0}\right| \geqq C_{1} N$, where $C_{1}$ is the same as in Lemma 2, and consider two regions

$$
\operatorname{Im} \lambda \geqq 0, \quad\left|\lambda-\lambda_{0}\right| \leqq 3 C_{1} N \quad \text { and } \quad \operatorname{Im} \lambda \leqq 0, \quad|\lambda|<\frac{1}{2} N
$$

We introduce the reduced kernel

$$
K_{N}\left(\lambda, \lambda_{0}\right)=e^{N\langle x\rangle_{N}}\left(\lambda^{2}-\lambda_{0}^{2}\right) R\left(\lambda_{0}\right) Q R_{0}(\lambda) e^{-N\langle x\rangle_{N}}
$$


and denote by $\mu_{j}(A)$ the characteristic values of the operator $A: L^{2} \rightarrow L^{2}$, that is, the eigenvalues of $\left(A A^{*}\right)^{\frac{1}{2}}$. When $\operatorname{Im} \lambda \geqq 0$ and $|\lambda| \leqq C N$ then

$$
\begin{aligned}
\mu_{j}\left(K_{N}\left(\lambda, \lambda_{0}\right)\right) \leqq & C N^{2} \mu_{j}\left(e^{N\langle x\rangle_{N}} R\left(\lambda_{0}\right) e^{-N\langle x\rangle_{N}-c\langle x\rangle}\right) \\
& \cdot\left\|e^{c\langle x\rangle+N\langle x\rangle_{N}} Q e^{N\langle x\rangle_{N}}(I+\Delta)^{-1}\right\| \\
& \times\left\|(I+\Delta) e^{-N\langle x\rangle_{N}} R_{0}(\lambda) e^{-N\langle x\rangle_{N}}\right\|,
\end{aligned}
$$

where the norms are defined by $\|\cdot\|=\|\cdot\|_{L^{2}\left(\mathbb{R}^{n}\right) \rightarrow L^{2}\left(\mathbb{R}^{n}\right)}$. By Lemma 1 and interpolation we get

$$
\left\|(I+\Delta) e^{-N\langle x\rangle_{N}} R_{0}(\lambda) e^{-N\langle x\rangle_{N}}\right\| \leqq C N^{1+\frac{1}{\gamma}} \max (1, N /(1+|\lambda|)),
$$

and by assumption (2.2)

$$
\left\|e^{c\langle x\rangle+N\langle x\rangle_{N}} Q e^{N\langle x\rangle_{N}}(I+\Delta)^{-1}\right\|=\mathcal{O}(1)
$$

independently of $N$. To estimate the characteristic values in (2.16) we introduce $\psi \in C_{0}^{\infty}(B(0,2)), 0 \leqq \psi \leqq 1, \psi \equiv 1$ in $B(0,1)$ and $\psi_{R}(x)=\psi\left(\frac{x}{R}\right)$ and let $\Delta_{R}$ be the Dirichlet Laplacian on $B(0.3 R)$. Then

$$
\begin{aligned}
\mu_{j}\left(e^{N\langle x\rangle_{N}} R\left(\lambda_{0}\right) e^{-N\langle x\rangle_{N}-c\langle x\rangle}\right) \leqq & \mu_{j}\left[\left(I+\Delta_{R}\right)^{-1}\left(I+\Delta_{R}\right) \psi_{R} e^{N\langle x\rangle_{N}} R\left(\lambda_{0}\right) e^{-N\langle x\rangle_{N}-c\langle x\rangle}\right] \\
& +\left\|\left(1-\psi_{R}\right) e^{-c\langle x\rangle+c\langle x\rangle+N\langle x\rangle_{N}} R\left(\lambda_{0}\right) e^{-N\langle x\rangle_{N}-c\langle x\rangle}\right\| \\
\leqq & \mu_{j}\left(\left(I+\Delta_{R}\right)^{-1}\right)\left\|e^{N\langle x\rangle_{N}} R\left(\lambda_{0}\right) e^{-N\langle x\rangle_{N}}\right\|_{H^{2}\left(\mathbb{R}^{n}\right) \rightarrow L^{2}\left(\mathbb{R}^{n}\right)} \\
& +C_{1} e^{-C R} \leqq C_{2}\left(R^{2} j^{-\frac{2}{n}}+e^{-C R}\right) .
\end{aligned}
$$

where we used Lemma 2 and the Weyl estimate on the number of eigenvalues of $\Delta_{R}$. Optimization in $R$ gives for $\operatorname{Im} \lambda \geqq 0,|\lambda| \leqq C N$,

$$
\mu_{J}\left(K_{N}\left(\lambda, \lambda_{0}\right)\right) \leqq C_{\delta} N^{3+\frac{1}{6}} \max (1, N /(1+|\lambda|)) j^{-\frac{2}{n+\delta}}
$$

for any $\delta>0$.

In the second region in $(2.14), \operatorname{Im} \lambda<0,|\lambda|<\frac{N}{2}$ we write

$$
\begin{aligned}
\mu_{J}\left(K_{N}\left(\lambda, \lambda_{0}\right)\right) & =\mu_{\jmath}\left(K_{N}\left(\lambda, \lambda_{0}\right)-K_{N}\left(-\lambda, \lambda_{0}\right)+K_{N}\left(-\lambda, \lambda_{0}\right)\right) \\
& \leqq \mu_{\jmath}\left(K_{N}\left(\lambda, \lambda_{0}\right)-K_{N}\left(-\lambda, \lambda_{0}\right)\right)+\mu_{J}\left(K_{N}\left(-\lambda, \lambda_{0}\right)\right),
\end{aligned}
$$

and we only need to estimate the first term on the right-hand side. We rewrite operator as

$$
e^{N\langle x\rangle_{N}}\left(\lambda^{2}-\lambda_{0}^{2}\right) R\left(\lambda_{0}\right) Q\left(R_{0}(\lambda)-R_{0}(-\lambda)\right) e^{-N\langle x\rangle_{N}}
$$

so that

$$
\mu_{j}\left(K_{N}\left(\lambda, \lambda_{0}\right)-K_{N}\left(-\lambda, \lambda_{0}\right)\right) \leqq C N^{2} \mu_{j}\left(e^{N\langle x\rangle_{N}} Q\left(R_{0}(\lambda)-R_{0}(-\lambda)\right) e^{-N\langle x\rangle_{N}}\right) .
$$


Using the spectral resolution of the Laplacian as in $[19,24,25]$ we now write $R_{0}(\lambda)-R_{0}(-\lambda)=E_{\lambda} E_{\hat{\lambda}}^{*}$, where $E_{\lambda}: L^{2}\left(S^{n-1}\right) \rightarrow e^{|\lambda|\langle x\rangle} L^{2}\left(\mathbb{R}^{n}\right)$ has the kernal $c \lambda^{\frac{n-2}{2}} e^{i \lambda\langle x, \omega\rangle}, \omega \in S^{n-1}$. If $\Delta_{S^{n-1}}$ is the Laplacian on $S^{n-1}$, it follows that

$$
\begin{aligned}
\mu_{j}\left(K_{N}\left(\lambda, \lambda_{0}\right)-K_{N}\left(-\lambda, \lambda_{0}\right)\right) \leqq & \left\|e^{N\langle x\rangle_{N}} Q e^{\frac{A}{2}\langle x\rangle^{1+\varepsilon}}(I+\Delta)^{-1}\right\| \\
& \times \mu_{j}\left((I+\Delta) e^{-\frac{A}{2}\langle x\rangle^{1+\varepsilon}} E_{\lambda}\left(I+\Delta_{S^{n-1}}\right)^{m}\left(I+\Delta_{S^{n-1}}\right)^{-m}\right) \\
& \times\left\|E_{\hat{\lambda}}^{*} e^{-N\langle x\rangle_{N}}\right\| \\
\leqq & C_{3}\left\|(I+\Delta) e^{-\frac{A}{2}\langle x\rangle^{1+\varepsilon}} E_{\lambda}\left(I+\Delta_{S^{n-1}}\right)^{m}\right\| \\
& \times \mu_{j}\left(\left(I+\Delta_{S^{n-1}}\right)^{-m}\right)\left\|E_{\lambda}^{*} e^{-N\langle x\rangle_{N}}\right\| .
\end{aligned}
$$

The two norms are estimated by Schur's Lemma:

$$
\sup _{x} e^{\delta\langle x\rangle}(1+|\lambda|)^{2+\frac{n-2}{2}} e^{-\frac{A}{2}\langle x\rangle^{1+x}+|\lambda|\langle x\rangle} C^{m}(2 m) ! \leqq \tilde{C} e^{\tilde{C}|\lambda|^{\frac{1+r}{\delta}}} C^{m}(2 m) !,
$$

where $e^{\delta\langle x\rangle}$ terms guarantees integrability in $x$, and

$$
\sup _{x} e^{\partial\langle x\rangle}|\lambda|^{\frac{n-2}{2}} e^{|\lambda|\langle x\rangle-N\langle x\rangle_{N}} \leqq \tilde{C} e^{\check{C} N^{\frac{1+\varepsilon}{r}}},
$$

since $|\lambda|<\frac{1}{2} N$. Hence

We now use the estimate $\mu_{j}\left(\left(I+\Delta_{S^{n-1}}\right)^{-m}\right) \leqq C j^{-\frac{2 m}{n-1}}$ and optimize in $m$.

$$
\mu_{j}\left(K_{N}\left(\lambda, \lambda_{0}\right)-K_{N}\left(-\lambda, \lambda_{0}\right)\right) \leqq C e^{C N^{\frac{1+\varepsilon}{\varepsilon}}-j^{\frac{1}{n-1} / C}},
$$

so that for $\operatorname{Im} \lambda \leqq 0,|\lambda|<\frac{1}{2} N$ we obtain

$$
\mu_{J}\left(K_{N}\left(\lambda, \lambda_{0}\right)\right) \leqq \begin{cases}C e^{C N^{1+\frac{1}{8}}}, & \text { if } j<\left(C N^{1+\frac{1}{\varepsilon}}\right)^{n-1} \\ C_{\delta} N^{3+\frac{1}{8}} \max (1, N /(1+|\lambda|)) j^{-\frac{2}{n+\delta}} & \text { if } j>\left(C N^{1+\frac{1}{\varepsilon}}\right)^{n-1}\end{cases}
$$

for any $\delta>0$.

By the proof of Proposition 1, the definition of $K_{N}\left(\lambda, \lambda_{0}\right)$. (2.15), and estimates (2.18) we see that for $\lambda$ in (2.14), the poles of $R(\lambda)$ are among the zeros of

$$
h_{N}(\lambda)=\underset{L^{2}\left(\mathbb{R}^{n}\right)}{\operatorname{det}}\left(I+\left(K_{N}\left(\lambda, \lambda_{0}\right)\right)^{n}\right)
$$

with multiplicity of the pole of $R(\lambda)$ less than or equal to the multiplicity of the corresponding zero of $h_{N}(\lambda)$ - see Appendices to [21 or 7].

Since $|\operatorname{det}(I+A)| \leqq \prod\left(1+\mu_{j}(A)\right)$ for $A$ trace class and since $\mu_{j}\left(B^{n}\right) \leqq$ $\left(\mu_{\left[\frac{1}{n}\right]}(B)\right)^{n}$ we can use $(2.18)$ to estimate $h_{N}(\lambda)$. If $\left|\lambda_{0}\right|=C_{1} N, \lambda_{0} \in i \mathbb{R}_{+}$we choose $c_{0}>0$ such that

$$
D\left(\lambda_{0}, C_{1} N+c_{0} N\right) \cap\{\lambda: \operatorname{Im} \lambda \leqq 0\} \subset D(0, N / 2),
$$




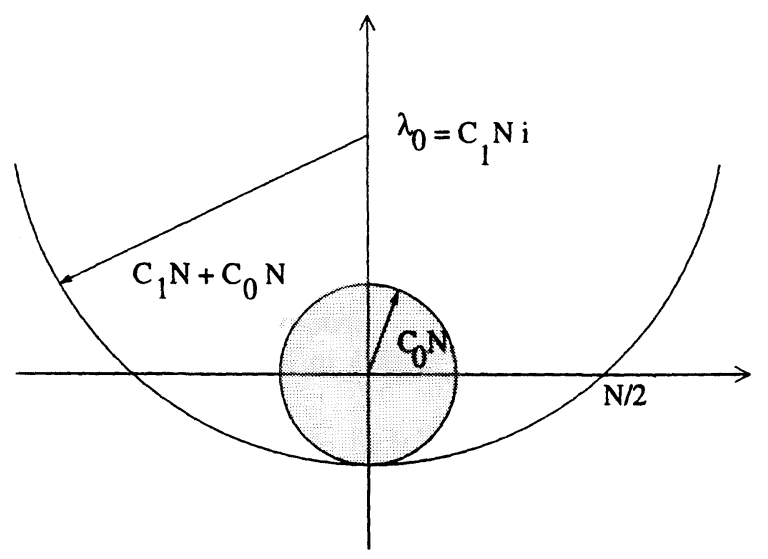

Fig. 2. Bound on the number of poles

in fact we can take $c_{0}<\sqrt{C_{1}^{2}+\frac{1}{4}}-C_{1}^{2}-$ see Fig. 2. For $\lambda \in \partial D\left(\lambda_{0}, 4 C_{1}+c_{0} N\right)$ (2.18) shows that

$$
\left|h_{N}(\lambda)\right| \leqq C_{\delta} e^{\left(C_{\delta} N^{\left(3+\frac{1}{\varepsilon}\right) \frac{n+2 \delta}{2}}+C\left(N^{\frac{1+t}{\varepsilon}}\right)^{n}\right)},
$$

since for any $\delta>0$

$$
\begin{gathered}
\prod_{1}^{\infty}\left(1+C_{\delta} N^{\left(3+\frac{1}{\delta}\right) n} j^{-2 \frac{n}{n+\delta}}\right) \leqq C_{\delta} e^{C_{\delta} N^{\left(3+\frac{1}{\varepsilon}\right) \frac{n+2 \delta}{2}}} \\
\prod_{1}^{C\left(N^{\frac{1+\varepsilon}{\varepsilon}}\right)^{n-1}}\left(1+C e^{C N^{\frac{1+\varepsilon}{r}}}\right) \leqq C e^{C\left(N^{\frac{1+r}{\delta}}\right)^{n}} .
\end{gathered}
$$

Since $h_{N}\left(\lambda_{0}\right)=1$, Jensen's inequality immediately bounds the number of zeroes of $h_{N}(\lambda)$ in $D\left(\lambda_{0}, C_{1} N+c_{0} N\right)$ by $C_{\delta} N^{n\left(\max \left(\frac{1+\varepsilon}{\varepsilon}, \frac{3}{2}+\frac{1}{2 \varepsilon}+\delta\right)\right)}$ for any $\delta>0$. Hence, we obtain a bound in $D\left(0, c_{0} N\right)$ (see Fig. 2) and that proves the proposition.

Remark. For our application in the next section we only need a polynomial bound. The expected "sharp" bound is $\mathcal{O}\left(r^{n\left(\frac{1+r}{r}\right)}\right)$ and $(2.13)$ gives that if $\varepsilon<1$. By refining the representation of the resolvent that improvement is very plausible as is a generalization to arbitrary compactly supported perturbations of operators satisfying (2.1) and (2.2) as has been initated for the Laplacian in [16]. We should remark however that the simple method above already gives the expected bound with the exponent $n(1+\varepsilon) / \varepsilon$ in the potential case. In fact, the operator $K_{N}\left(\lambda, \lambda_{0}\right)$ can then be replaced by $e^{N\langle x\rangle_{N}} V R_{0}(\lambda) \varepsilon^{-N\langle x\rangle_{N}}$ which reduces the power $3+\frac{1}{\varepsilon}$ to $1+\frac{1}{\varepsilon}$ in (2.17), but the modified proof of Proposition 1 requires now that (2.2) holds for $\partial^{\alpha} V,|\alpha| \leqq 2$. This gives the desired improvement. We also note that when $n=1$ and $\frac{1+\varepsilon}{\varepsilon} \notin \mathbb{Z}$, the bound is optimal as can be seen by the methods of [23]. In fact, Froese [5], using a different method, proved recently asymptotic formulae for the number of poles for a class of non-compactly supported potentials, including some cases where $\frac{1+\varepsilon}{\varepsilon} \in \mathbb{Z}$. 


\section{Generalized Poisson Formula}

We will now apply the estimate (2.13) of Sect. 2 to generalize Melrose's Poisson formula $[11,18]$ to a class of non-compactly supported perturbations. Although we consider only operatoes $P$ satisfying the assumptions of Theorem in Sect. 1 it will be clear from the simple argument in the proof of proposition 3 that further generalization would follow from extending Proposition 2 to other perturbation and relaxing regularity assumptions.

We start by introducing a family of compactly supported perturbations of $\Delta$. Let $\psi \in C_{0}^{\infty}(B(0,2))$ satisfy $0 \leqq \psi \leqq 1, \psi \equiv 1$ in $B(0,1)$ and let $\psi_{R}(x)=\psi(x / R)$. We then consider

$$
P_{R}=\psi_{R} P+\left(1-\psi_{R}\right) \Delta \text {. }
$$

If $R$ is large enough then $P_{R}$ is an elliptic compactly supported perturbation of $\Delta$ on $\mathbb{R}^{n}$. Let us denote by $U_{R}(t)$ the wave group for $D_{t}^{2}-P_{R}$ and by $\left\{\lambda_{j}^{R}\right\}$ the resonances of $P_{R}$ (that is, poles of the meromorphic continuation of the resolvent $\left(P_{R}-\lambda^{2}\right)^{-1}$ included according to their multiplicity). The standard observation based on partial hypoellipticity (see [11 or 18, Lemma 3.1]) shows that $U_{R}(t)-U_{0}(t)$ is trace class in the sense of distributions. Here $U_{0}(t)$ is the trace wave group associated to $D_{t}^{2}-\Delta$.

We will need two continuity results as $R \rightarrow \infty$ both of which are essentially well-known but we outline the proofs for the sake of completeness.

Lemma 3. The operator $U(t)-U_{0}(t)$ is trace class as a distribution in $t$ and

$$
\operatorname{tr}\left(U_{R}(t)-U_{0}(t)\right) \underset{R \rightarrow \infty}{\stackrel{\mathscr{C}^{\prime}(\mathbb{R})}{\longrightarrow}} \operatorname{tr}\left(U(t)-U_{0}(t)\right) .
$$

Proof. We recall that

$$
U_{0}(t)=e^{i t A_{0}}, \quad U(t)=e^{i t A},
$$

where

$$
A_{0}=\left(\begin{array}{cc}
0 & I \\
\Delta & 0
\end{array}\right), \quad A=\left(\begin{array}{cc}
0 & I \\
P & 0
\end{array}\right)
$$

acting on $H^{1}\left(\mathbb{R}^{n}\right) \oplus L^{2}\left(\mathbb{R}^{n}\right)$ (see Sect. 2 of $\left.[18]\right)$. Hence

$$
U(t)-U_{0}(t)=\int_{0}^{t} U_{0}(t-s)\left(A-A_{0}\right) U(s) d s .
$$

Also, for $|r|<T, e^{M\langle x\rangle} U_{0}(r) e^{-M\langle y\rangle}=\mathcal{O}_{T, M}(1): H^{s+1}\left(\mathbb{R}^{n}\right) \oplus H^{s}\left(\mathbb{R}^{n}\right) \rightarrow H^{s+1}\left(\mathbb{R}^{n}\right)$ $\oplus H^{s}\left(\mathbb{R}^{n}\right)$ by the Huyghens principle. This and the partial hypoellipticity of $D_{t}^{2}-P$ and $D_{t}^{2}-\Delta$ (see e.g. Lemma 3.1 of [18]) show that for $\rho \in C_{0}^{\infty}(\mathbb{R})$ and any $M$ and $s$,

$$
\int\left(U(t)-U_{0}(t)\right) \rho(t): \dot{H}^{1}\left(\mathbb{R}^{n}\right) \oplus L^{2}\left(\mathbb{R}^{n}\right) \rightarrow e^{-M\langle x\rangle} H^{s+1}\left(\mathbb{R}^{n}\right) \oplus e^{-M\langle x\rangle} H^{s}\left(\mathbb{R}^{n}\right) .
$$

and hence the operator is trace class.

To consider the limit (3.2) we note that

$$
\frac{d}{d R} \operatorname{tr}\left(U_{R}(t)-U_{0}(t)\right)=-R^{-1} t \operatorname{tr}\left(\psi_{R}^{\prime} Q \frac{\sin t \sqrt{P_{R}}}{\sqrt{P_{R}}}\right),
$$


where $\psi_{R}^{\prime}(x)=\psi(x / R)$. Hence, for $\rho \in C_{0}^{\infty}(\mathbb{R})$

$$
\begin{aligned}
& R\left|\frac{d}{d R}\left(\operatorname{tr} \int\left(U_{R}(t)-U_{0}(t)\right) \rho(t) d t\right)\right| \\
& \leqq\left\|\psi_{R}^{\prime} Q\left(\int \frac{\sin t \sqrt{P_{R}}}{\sqrt{P_{R}}} \cdot t \rho(t) d t\right)\right\|_{\mathscr{L}^{\prime}\left(L^{2}\left(\mathbb{R}^{n}\right), L^{2}\left(\mathbb{R}^{n}\right)\right)} \\
& \leqq\left\|\mathbb{1}_{|x|>R} Q e^{\frac{A}{2}|x|^{1+\varepsilon}}(I+\Delta)^{-1}\right\|_{\mathscr{L}\left(L^{2}\left(\mathbb{R}^{n}\right), L^{2}\left(\mathbb{R}^{n}\right)\right)} \\
& \quad \times\left\|(I+\Delta) e^{-\frac{A}{2}|x|^{1+r}} \psi_{R}^{\prime} \int \frac{\sin t \sqrt{P_{R}}}{\sqrt{P_{R}}} t \rho(t) d t\right\|_{\mathscr{L}\left(L^{2}\left(\mathbb{R}^{n}\right), L^{2}\left(\mathbb{R}^{n}\right)\right)}
\end{aligned}
$$

The last trace class norm is uniformly bounded in $R$ (since $\int P_{R}^{-1 / 2} \sin t P_{R}^{\frac{1}{2}} t \rho(t) d t$ is uniformly bounded from $L^{2}\left(\mathbb{R}^{n}\right)$ to $\left.H^{s}\left(\mathbb{R}^{n}\right)\right)$. Hence the derivative of the trace is rapidly decaying and (3.2) follows by integration.

Lemma 4. Let $T>0$ be such that no scattering pole of $P$ has modulus $T$. Let $\left\{\mu_{j}\right\}_{j=1}^{m_{T}}$ be the scattering poles of $P$ in $D(0, T)$ included according to their multiplicities. Then there exists a constant $R(T)$ such that for $R>R(T)$ the poles of $P_{R}$ in $D(0, T)$ are given by $\left\{\mu_{j}^{R}\right\}_{j=1}^{m_{T}}$ and $\mu_{j}^{R} \rightarrow \mu_{j}$ as $R \rightarrow \infty$.

Proof. Again for the reader's convenience we outline the modification required by non-compact support (see [13] for the compact case and also Theorem 3.1 in Chapter 1 of [8]). Let $R_{R}(\lambda)$ denote the meromorphic continuation of the resolvent $\left(P_{R}-\lambda^{2}\right)^{-1}$ and let $r(T)$ be such that

$$
D\left(\mu_{\jmath}, r(T)\right) \subset D(0, T), \quad D\left(\mu_{i}, r(T)\right) \cap D\left(\mu_{\jmath}, r(T)\right)=\phi, \quad \text { if } \mu_{l} \neq \mu_{j} .
$$

Then for $R$ large enough (so that $\left\|P_{R}-P\right\|_{e^{T\langle x\rangle} H^{2} \rightarrow e^{-T\langle x\rangle} L^{2}}$ is small enough depending on $T$ ) we have

$$
R_{R}(\lambda)=R(\lambda)\left(I+\left(P_{R}-P\right) R(\lambda)\right)^{-1}, \quad \lambda \in D(0, T) \backslash \bigcup_{j=1}^{m_{T}} D\left(\left(\mu_{j}, r(T)\right)\right)
$$

as operators $e^{-T\langle x\rangle} L^{2} \rightarrow e^{T\langle x\rangle} L^{2}$. The continuity of the poles follows.

We can now prove

Proposition 3. Let $P$ be an operator satisfying the assumptions of Theorem in Sect. 1. If $U(t)$ is the wave group for $D_{t}^{2}-P, U_{0}(t)$ the free wave group and $\left\{\lambda_{j}\right\}$ the set of poles of the meromorphic continuation of $\left(P-\lambda^{2}\right)^{-1}$ (included according to their multiplicities) then

$$
\operatorname{tr}\left(U(t)-U_{0}(t)\right)=\sum e^{\mp i \lambda_{j} t}, \quad \pm t>0
$$

in the sense of distributions.

Proof. Let $\varphi \in C_{0}^{\infty}\left(\mathbb{R}_{+}\right)$. By Lemma 3 and Melrose's Poisson formula for compactly supported perturbations (see Theorem 3 of [18]) it suffices to show that

$$
\left\langle\sum e^{-i i^{R}, t}, \varphi\right\rangle \underset{R \rightarrow \infty}{\longrightarrow}\left\langle\sum e^{-i \lambda, t}, \varphi\right\rangle
$$

that is $\sum \hat{\varphi}\left(\lambda_{1}^{R}\right) \underset{R \rightarrow \infty}{\rightarrow} \sum \hat{\varphi}\left(\lambda_{j}\right)$. 
Since the upper bound in Proposition 2 is uniform with respect to $R$,

$$
\#\left\{\lambda_{j}^{R}:\left|\lambda_{j}^{R}\right| \leqq r\right\} \leqq C r^{N},
$$

we use the Paley-Wiener estimate $|\hat{\varphi}(\lambda)| \leqq C_{M}(1+|\lambda|)^{-M}, \operatorname{Im} \lambda \leqq 0$, any $M$, to decompose the sums as follows:

$$
\begin{aligned}
\sum \hat{\varphi}\left(\lambda_{j}^{R}\right) & =\sum_{\left|\lambda_{j}^{R}\right| \leqq T} \hat{\varphi}\left(\lambda_{j}^{R}\right)+\mathcal{O}\left(\sum_{\left|\lambda_{j}^{R}\right| \geqq T} C_{M}\left(1+\left|\lambda_{j}\right|\right)^{-M}\right) \\
& =\sum_{\left|\lambda_{j}^{R}\right| \leqq T} \hat{\varphi}\left(\lambda_{j}^{R}\right)+\mathcal{O}_{M}\left(T^{-M+N}\right)
\end{aligned}
$$

and

$$
\sum \hat{\varphi}\left(\lambda_{j}\right)=\sum_{\left|\lambda_{j}\right| \leqq T} \hat{\varphi}\left(\lambda_{j}\right)+\mathcal{O}_{M}\left(T^{-M+N}\right)
$$

for any $M$. We now choose a sequence $T_{k} \rightarrow \infty$ such that for each $T=T_{k}$ the assumptions of Lemma 4 are satisfied. Since then $\sum_{\left|\lambda_{j}\right| \leqq T} \hat{\varphi}\left(\lambda_{j}\right)=\sum_{\left|\lambda_{j}^{R}\right| \leqq T} \hat{\varphi}\left(\lambda_{j}^{R}\right)+$ $o_{T}(1)$ as $R \rightarrow \infty$, we get

$$
\sum \hat{\varphi}\left(\lambda_{j}\right)=\sum \hat{\varphi}\left(\lambda_{j}^{R}\right)+o_{T}(1)+\mathcal{O}_{M}\left(T^{-M+N}\right)
$$

as $R \rightarrow \infty$ and consequently

$$
\sum \hat{\varphi}\left(\lambda_{j}\right)=\lim _{R \rightarrow \infty} \sum \hat{\varphi}\left(\lambda_{j}^{R}\right) .
$$

\section{Existence of Resonances}

As was noted in [18] lower bounds for resonances can be obtained from the singularities of $\operatorname{tr}\left(U(t)-U_{0}(t)\right)$ which "spread out" from 0 , that is are not $\delta$-functions. A weaker immediate statement is

$$
\operatorname{tr}\left(U(t)-U_{0}(t)\right) \neq 0 t>0 \Rightarrow \text { there exists at least one resonance or eigenvalue }
$$

and that conclusion is sufficient for the Corollary in Sect. 1.

When $P$ is an elliptic operator with $C^{\infty}$ coefficients on $\mathbb{R}^{n} \backslash \mathcal{O}, \mathcal{O} \subset \mathbb{R}^{n}$ bounded, $\partial \mathcal{O}$ smooth, then $\operatorname{tr}\left(U(t)-U_{0}(t)\right)$ has a full asymptotic expansion at $t=0$ and when $\mathcal{O} \neq \phi$, Example 5.2 of [18] gave a lower bound $r^{n-1-\varepsilon}$, any $\varepsilon>0$, for the number of poles. In greater generality, assume that $\operatorname{tr}\left(U(t)-U_{0}(t)\right)$ has a regular asymptotic expansion at $t=0$ :

$$
\begin{gathered}
\operatorname{tr}\left(U(t)-U_{0}(t)\right) \sim \mathcal{c}_{-n}(P) D_{t}^{n-1} \delta_{0}(t)+c_{-n+1}(P)\left|D_{t}\right|^{n-2} \delta_{0}(t) \\
+\cdots+c_{0}(P)+c_{1}(P)|t|+\cdots .
\end{gathered}
$$

Lemma 5. If $c_{0}(P)=0$ and $c_{k}(P) \neq 0$ for some $k>0$ in (4.2) then there exists infinitely many scattering poles.

Proof. Since the non-vanishing of $c_{k}(P)$ clearly gives the existence of resonances or eigenvalues we only need to check that there are infinitely many - there can only be finitely many eigenvalues. Otherwise however the value at $t=0$ would be the number of $\lambda_{J}$ 's, and since $c_{0}(P)=0$ that is impossible. 
In dimension three the now standard procedure of computing the wave/heat coefficients using the Hadamard parametrix constructions gives

Lemma 6. If $n$ is odd and $P=\Delta_{g}+V$ then $c_{2 k}(P)=0$ and if $n=3$ then

$$
c_{1}(P)=a \int\left(\frac{1}{360}\left(6|\mathrm{Ric}|^{2}+3 \tau^{2}\right)-\frac{1}{6} V \tau+\frac{1}{2} V^{2}\right) d \operatorname{vol}_{y}, \quad a \neq 0
$$

where Ric is the Ricci curvature tensor of $g$ and $\tau$ its scalar curvature.

Proof. We shall briefly outline the argument using heavily the notation from [1] where the computation is done for $\Delta_{g}$ on a compact manifold. The computation reduces to that case by compactification and continuity. In fact, we can approximate $\operatorname{tr}\left(U(t)-U_{0}(t)\right)$ by $\operatorname{tr}\left(U_{R}(t)-U_{0}(t)\right)$ using Lemma 3 and then can observe that $\operatorname{tr}\left(U_{R}(t)-U_{0}(t)\right)$ is equal, for $t$ small, to the trace of a self adjoint reference operator on a torus (see Sect. 5 of [18]). The assumption of the decay of coefficients and the boundedness of their derivative give, by interpolation, exponential decay of all derivatives. This is much stronger than the standard short range conditions (see [14]) needed for the convergence of all the heat/wave coefficients. Thus we need to study the operator $P=\Delta_{g}+V, V \in C^{\infty}(M)$, on a compact three dimensional manifold, $(M, g)$. From Sect. 28 of [1] we have

$$
\begin{aligned}
\cos t \sqrt{P}(x, y)= & C_{0}\left(u_{0}^{V}(x, y)|t| \delta^{\prime}\left(\overline{x y}^{2}-t^{2}\right)\right. \\
& -\frac{1}{4} u_{1}^{V}(x, y)|t| \delta\left(\overline{x y}^{2}-t^{2}\right) \\
& \left.+\frac{1}{4^{2}} u_{2}^{V}(x, y)|t|\left(\overline{x y}{ }^{2}-t^{2}\right)_{-}^{0}+\cdots\right),
\end{aligned}
$$

where $\overline{x y}$ denotes the distance between $x$ and $y, u_{k}^{V}$ satisfy a modification of transport equations (9) in [1] giving a modification of (10) there:

$$
\begin{aligned}
u_{0}^{V}(x, y) & =\Theta^{-\frac{1}{2}}(x, y) \\
u_{k+1}^{V}(x, y) & =\Theta^{-\frac{1}{2}}(x, y) \int_{0}^{1} s^{k} \Theta^{\frac{1}{2}}\left(x, x_{s}\right)\left(\Delta_{2}+V\left(x_{s}\right)\right) u_{k}^{V}\left(x, x_{s}\right) d s,
\end{aligned}
$$

where $x_{s}=\exp _{x} s v, \quad x_{1}=y, \quad \Theta(x, y)=\left|\operatorname{det}_{y} T_{\exp _{r}^{-1}(y)} \exp _{x}\right|$ and $\Delta_{2}$ denotes the Laplacian applied in the second variable. We are interested in computing $\int_{M} u_{2}^{V}(x, x) d \operatorname{vol}_{y}$. From (4.4) we obtain the expression of $u_{k}^{V}$ in terms of $u_{k}=u_{k}^{0}$ of $[1]$ :

$$
\begin{aligned}
u_{1}^{V}(x, y)= & u_{1}(x, y)+\Theta^{-\frac{1}{2}}(x, y) \int_{0}^{1} V\left(x_{s}\right) d s \\
u_{2}^{V}(x, y)= & u_{2}(x, y)+\Theta^{-\frac{1}{2}}(x, y) \int_{0}^{1} s \Theta^{\frac{1}{2}}\left(x, x_{s}\right) V\left(x_{s}\right) u_{1}\left(x, x_{s}\right) d s \\
& +\Theta^{-\frac{1}{2}}(x, y) \int_{0}^{1} s \Theta^{\frac{1}{2}}\left(x, x_{s}\right)\left(\Delta_{2}+V\left(x_{s}\right)\right) \\
& \times\left(\Theta^{-\frac{1}{2}}\left(x, x_{s}\right) \int_{0}^{1} v\left(x_{s^{\prime}}(s)\right) d s^{\prime}\right) d s,
\end{aligned}
$$


where $x_{s^{\prime}}(s)=\exp _{x} s^{\prime} w, x_{1}(s)=x_{s}$. When $x=y$ we have $x_{s}=x$ and we only need to check the value of $\left.\Delta_{2}\left(\Theta^{-\frac{1}{2}}\left(x, x_{s}\right) \int_{0}^{1} V\left(x_{s^{\prime}}(s)\right) d s^{\prime}\right)\right|_{x_{s}=x}$. Since $\Theta(x, y)-1$ vanishes quadratically at $x=y$, we get $($ see $(4.4)) u_{1}(x, x)+\left.\int_{0}^{1} \Delta_{z} V\left(\exp _{z}\left(1-s^{\prime}\right) w\right)\right|_{z=x} d s^{\prime}$, where $\exp _{z} w=x$. In normal geodesic coordinates centered at $z$ (identifying $M$ with $\left.T_{z} M\right)$ we have $\exp _{z}\left(1-s^{\prime}\right) w=\left(1-s^{\prime}\right)(x-z)$ and $\Delta_{z}=-\sum \partial_{z}^{2} \bmod \mathcal{O}(x-$ $z)$ Diff ${ }^{2}$. Writing $\quad V\left(\exp _{z}\left(1-s^{\prime}\right) w\right)=V(z)+\left(1-s^{\prime}\right) \nabla V(z)(x-z)+\left(1-s^{\prime}\right)^{2}$ $\langle\operatorname{Hess} V(x-z), x-z\rangle / 2+\mathcal{O}(x-z)^{3}$, we obtain

$$
\left.\Delta_{z} V\left(\exp _{z}\left(1-s^{\prime}\right) w\right)\right|_{z=x}=\Delta V(x)-2\left(1-s^{\prime}\right) \Delta V(x)+\left(1-s^{\prime}\right)^{2} \Delta V(x)
$$

Returning to (4.5) we see that

$$
u_{2}^{V}(x, x)=u_{2}+V(x) u_{1}(x, x)+\frac{1}{2} V(x)^{2}+\frac{1}{6} \Delta V(x) .
$$

After the integration over $M$ the last term disappears and Sect. 24 of [1] gives the lemma.

We can now prove Theorem of Sect. 1. For that we only need $c_{1}(P) \neq 0$ and that is obvious from Lemma 6 if $R \equiv 0$ and $V \neq 0$. Hence we only need to consider the non-flat case. Since $\tau=\operatorname{tr}$ Ric, the Cauchy-Schwartz inequality shows, in dimension three, $3 \mid$ Ric $\left.\right|^{2} \geqq \tau^{2}$ with equality only if

$$
\operatorname{Ric}=(1 / 3) \tau I d
$$

If this were true at every point of $M$, then by Theorem 1.97 of [2], the scalar curvature $\tau$ (and in fact by Proposition 1.20 there also the sectional one) would be constant which is impossible here as $\tau(x) \rightarrow 0$ as $|x| \rightarrow \infty$ and the metric is not flat. Hence

$$
c_{1}(P) / a>\int\left(\frac{1}{72} \tau^{2}-\frac{1}{6} V \tau+\frac{1}{2} V^{2}\right) d \operatorname{vol}_{\mathfrak{y}} .
$$

Since $4(1 / 72)(1 / 2)=(1 / 6)^{2}$ the expression on the right is positive semi-definite and consequently we obtain the needed fact that $c_{1}(P) \neq 0$.

Remark. Since our result depends on the exact values of the constants in Lemma 6, to gain the reader's confidence, we will verify it for the case of the three sphere (see also the comment after the Corollary in Sect. 1). We put $V=\tau / 6=1$ so that the eigenvalues of $P=\Delta+V$ are $(k+1)^{2}$ with multiplicities $(k+1)^{2}, k=$ $0,1, \ldots$. Hence $\operatorname{tr} \cos t \sqrt{\Delta+V}=\frac{1}{2} \sum_{n \in \mathbb{Z}} n^{2} e^{i n t}$ which near $t=0$ is a multiple of $\delta_{0}^{\prime \prime}(t)$. Hence $c_{1}(P)=0$ as putting $V=\tau / 6$ and $|\mathrm{Ric}|^{2}=\tau^{2} / 3$ (since in that case (4.6) clearly holds) in Lemma 6 shows.

Acknowledgements. This paper is a result of a conversion with Richard Melrose who, after we observed directly existence of infinitely many resonances for fixed sign potentials, pointed out special properties of heat coefficients in three dimensions. We would like to thank him and Steve Zelditch for helpful discussions and the National Science Foundation for partial support under grants DMS-9202361 and DMS-9202344. The first author was also supported by an Alfred P. Sloan fellowship. 


\section{References}

1. Bérard, P.: On the wave equation on a compact manifold without conjugate points. Math. Z. 155, 249-276 (1977)

2. Besse, A.: Einstein Manifolds. Berlin, Heidelberg, New York: Springer, 1986

3. Deift, P.A.: Application of a commutation formula. Duke Math. J. 45, 267-310 (1978)

4. Farhy, L.: Distribution near the real axis of scattering poles generated by a non-hyperbolic periodic ray (To appear in Ann. Inst. H. Poincaré)

5. Froese, R.: Asymptotic distribution of resonances in one dimension. Preprint, 1994

6. Guillopé, L.: Majorations optimales pour le nombre de résonance d'une perturbation compacte du laplacien euclidien. Preprint, 1993

7. Guillopé, L., Zworski, M.: Polynomial bounds on the number of resonances for some complete spaces of constant negative curvature near infinity. To appear in Asymp. Anal.

8. Gohberg, I., Krein, M.: Introduction to the theory of non-self-adjoint operators. Providence, RI: AMS 1969

9. Melrose, R.B.: Scattering theory and the trace of the wave group. J. Funct. Anal. 45, 29-40 (1982)

10. Melrose, R.B.: Polynomial bounds on the number of scattering poles. J. Funct. Anal. 53, 287-303 (1983)

11. Melrose, R.B.: Polynomial bounds on the distribution of poles in scattering by an obstacle. Journées "Équations aux Dérivées partielles", Saint-Jean de Monts, 1984

12. Melrose, R.B.: Lectures at Stanford. Geometric scattering theory. Cambridge: Cambridge University Press (To appear)

13. Petras, S.V.: On the continuous dependence of the poles of the scattering matrix on the coefficients of an elliptic operator. Proc. Steklov Inst. Math. 159, 135-139 (1983)

14. Robert, D.: Asymptotique de la phase de diffusion à haute énergie pour des perturbations du second orders du Laplacien. Ann. Scient. Éc. Norm. Sup. $4^{e}$ série, t. 25, 107-134 (1992)

15. Shubin, M., Sjöstrand, J.: Appendice à l'exposé: Weak Bloch property and weight estimates for elliptic operators. Séminaire Équations aux Dérivées Partielles, Ecole Polytechnique, 19891990

16. Sjöstrand, J., Zworski, M.: Complex scaling and the distribution of scattering poles. J. Amer. Math. Soc. 4(4), 729-769 (1991)

17. Sjöstrand, J., Zworski, M.: Lower bounds on the number of scattering poles. Comm. P.D.E. 18, 847-858 (1993)

18. Sjöstrand, J., Zworski, M.: Lower bounds on the number of scattering poles II. J. Funct. Anal. 123(2), 336-367 (1994)

19. Vodev, G.: Sharp polynomial bounds on the number of scattering poles for metric perturbations of the Laplacian in $\mathbf{R}^{n}$. Math. Ann. 291, 39-49 (1991)

20. Vodev, G.: Sharp polynomial bounds on the number of scattering poles for perturbations of the Laplacian. Commun. Math. Phys. 146, 205-216 (1992)

21. Vodev, G.: Sharp bounds on the number of scattering poles in even dimensional spaces. Duke Math. J. 74, 1-17 (1994)

22. Vodev, G.: Asymptotics for the number of scattering poles. To appear in J. Funct. Anal

23. Zworski, M.: Distribution of scattering poles for scattering on the real line. J. Funct. Anal. 73(2), 277-296 (1987)

24. Zworski, M.: Sharp polynomial bounds on the number of scattering poles. Duke Math. J. 59(2), 311-323 (1989)

25. Zworski, M.: Counting scattering poles. Spectral and Scattering Theory. Ikawa, M. (ed.) New York: Marcel Dekker, 1994 
